\title{
Altering the $\mathrm{N}$-terminal arms of the polymerase manager protein UmuD modulates protein interactions
}

David A. Murison, Jaylene N. Ollivierre, Qiuying Huang, David E. Budil, and Penny J. Beuning
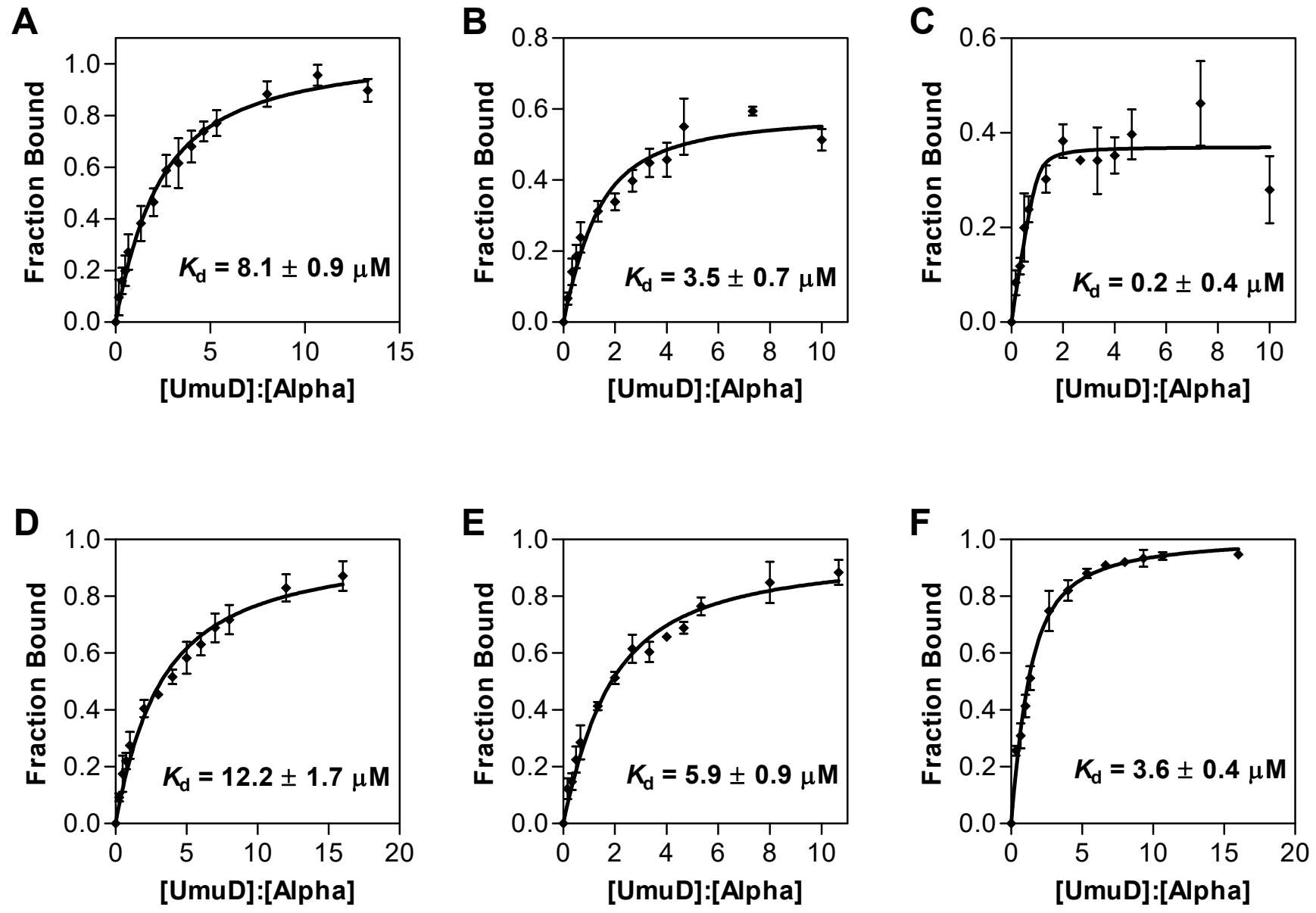

Supplemental Fig. 2. UmuD 8-S60A protein interacts with the Alpha Subunit of DNA

Polymerase III at the N-terminal PHP domain. Binding constants were determined by tryptophan fluorescence for (A) UmuD 8 S60A and Alpha WT, (B) UmuD 8 S60A and Alpha1-280, (C) UmuD 8 S60A and Alpha917-1160, (D) UmuD 18 S60A and Alpha WT, (E) UmuD 18 S60A and Alpha1-280, and $(F)$ UmuD18 S60A and Alpha917-1160. Curves represent the fraction of Alpha fluorescence quenched by increasing concentrations of UmuD protein which produced the $K_{d}$ values shown. 